\title{
Symptomatic Myocardial Bridging or Cardiac Whipple's Disease
}

\author{
Virgínia L. Ribeiro Cabral, Daniella Knecht, Regina Célia Pego, Nuno C. Figueiredo Silva
}

São Paulo, SP - Brazil

This report concerns a patient with articular and cardiac manifestations of Whipple's disease. The disease was diagnosed only when gastrointestinal symptoms had appeared, because all cardiac symptoms were attributed exclusively to myocardial bridging. After 18 months of treatment with trimethoprim-sulfamethoxazole, the patient is fully asymptomatic with a normal echocardiogram.

Whipple's disease is a rare, chronic systemic disease caused by a recently identified bacillus, Tropheryma whippelii ${ }^{1}$. More than $80 \%$ of the affected patients are white males. Its classic clinical presentation includes chronic diarrhea, malabsorption, and weight loss; however, these symptoms may appear late in the course of the disease. Symptoms related to involvement of other organs, such as the heart, lungs, brain, joints, lymph nodes, eyes, and skin, without gastrointestinal manifestations often delay the diagnosis ${ }^{2}$. The diagnosis is based on the presence of macrophages in lamina propria of affected tissues containing granules positive on periodic acid Schiff (PAS) staining and electron microscopy showing intra- and extracellular bacilli ${ }^{3}$. Recently, Tropheryma whippelii have been detected in peripheral blood mononuclear cells by polymerase chain reaction $(\mathrm{PCR})^{4}$.

\section{Case Report}

The case of 50-year-old man whose cardiac symptoms were attributed exclusively to myocardial bridging is presented. His disease started 6 years ago, with migratory nondeforming seronegative polyarthritis associated with axial involvement, which spontaneously resolved without any treatment. One year after the onset of joint symptoms, the

Hospital Heliópolis - São Paulo

Mailing address: Virgínia L. Ribeiro Cabral - Rua Rafael Sampaio Vidal, 77 09550-170 - São Caetano do Sul, SP - Brazil - E-mail: jalfeu@terra.com.br patient suffered from progressive dyspnea, palpitations, and precordial pain. In fact, he was admitted to the hospital with a diagnosis of coronary disease and atrial flutter. Serum cardiac enzymes and routine laboratory tests were considered normal, except for mild, microcytic anemia. He underwent electrical cardioversion, and amiodarone was started. Echocardiography revealed mild antero-lateral wall hypokinesis, a moderate left ventricular dilatation with preserved ejection fraction, thickening of the mitral and tricuspid cusps, aortic and mitral regurgitation, and moderate pericardial effusion. Electrocardiographic monitoring showed supraventricular tachycardia, and cardiac catheterization demonstrated normal coronary arteries and myocardial bridging in the left anterior descending coronary artery. He remained asymptomatic for a long period, although repeated echocardiography was consistent with the previous findings.

He was readmitted to the hospital because of a 10month history of liquid, diarrhea, malnutrition, and anorexia. Previously he had undergone a colonoscopy with rectum biopsy, showing a nonspecific chronic colitis that was treated with sulfasalazine and corticosteroids without any clinical improvement. His blood pressure was $90 / 60 \mathrm{mmHg}$, and nonthrombocytopenic purpura was noted on both legs. On cardiac auscultation, a grade II pansystolic murmur was detected at the apex. Laboratory examination showed a hemoglobin of $8.8 \mathrm{~g} / \mathrm{dL}$, mean corpuscular volume of $65 \mathrm{fL}$, normal ferritin level, an erythrocyte sedimentation rate (ESR) of $59 \mathrm{~mm} / \mathrm{h}$, platelet count of $480,000 \mathrm{~mm}^{3}$, and serum albumin of $29 \mathrm{~g} / \mathrm{L}$. A barium transit of the small bowel was normal, colonoscopy detected hyperemia in the left colon, and the biopsy showed a normal colonic mucosa. Abdominal computed tomography (CT) demonstrated thickened intestinal walls. At upper gastrointestinal endoscopy, duodenal mucosa appeared hyperemic with petechiae. Histopathological examination detected infiltration of the lamina propria by PAS-positive and Ziehl-Neelsen-negative macrophages. Oral therapy with trimethoprim-sulfamethoxazole and folate were started and continued for 18 months. All clinical signs of malabsorption disappeared, previous 


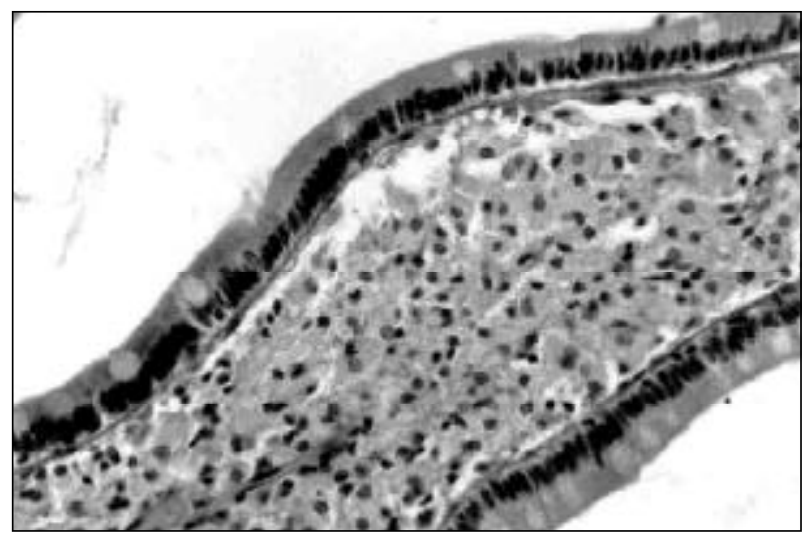

Fig. 1 - Duodenal biopsy (hematoxylin-eosin stain)- numerous foamy macrophages with granular cytoplasm in lamina propria.

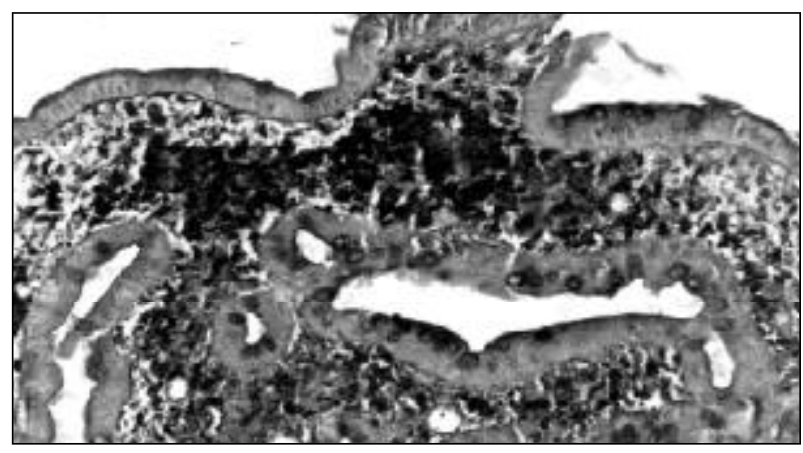

Fig. 2 - Duodenal biopsy (PAS stain) - marked infiltration of the lamina propria with PAS- positive macrophages.

abnormal laboratory findings returned to normal, and echocardiography showed resolution of cardiac abnormalities. Six months after discontinuing treatment, the patient is well without relapse.

\section{Discussion}

Whipple's disease has a variable clinical presentation. When no intestinal symptom occurs, it is often diagnosed late in its course. This disease occurs frequently in white men, between the 4 th and 6 th decades of life. A predominance of cases are described in the United States and Europe. The prevalence and demographic features of this disease in Brazil are not known. In this case, the digestive symptoms were preceded for 6 years by arthralgias. At the onset, a mild anemia was detected and cardiac symptoms were attributed to the presence of a myocardial bridging. It is characterized by systolic compression of a portion of the coronary artery by a segment of overlying myocardium. The muscle bridges commonly involves the left anterior descending coronary artery and in most cases evolves in a benign manner ${ }^{5}$, but some authors have reported this condition in association with angina pectoris, myocardial infarction, arrhythmias, and sudden death ${ }^{6,7}$. In this case, anemia, pericardial effusion, and valvular deformities could not be attributed to this anatomic anomaly. A possible causal relationship between Whipple's disease and cardiac involvement was the resolution of echocardiography features after antibiotic therapy. A review of the literature demonstrates the high prevalence of cardiac manifestations in patients with Whipple's disease. It is now recognized that valvar deformities in a patient suffering from Whipple's disease are caused by the disease and are not because of coexistent rheumatic heart disease as it had been believed ${ }^{8}$.

In Whipple's disease, the 3 cardiac layers may be attacked. The pericardium is frequently affected by small asymptomatic effusion or fibrinous pericarditis. Myocarditis, conduction tissue defects, or both, with identification of bacilli in the atrioventricular node and His bundle have been documented ${ }^{9}$. Endocardial involvement includes valve lesions or even the presence of vegetation, frequently erroneously diagnosed as classic bacterial endocarditis ${ }^{2,10-12}$. Another cardiac manifestation occurs because of the involvement of coronary arteries, where the identification of bacilli in the tunica media may be associated with focal ischemic degeneration of myocardium ${ }^{13}$. This reinforces the hypothesis that various focal infections can be one cause of progressive narrowing of coronary arteries. It is interesting to discuss whether the symptoms of myocardial ischemia experienced by this patient can be attributed exclusively to myocardial bridging. It should be emphasized that Whipple's disease may exist without gastrointestinal symptoms. If this happens, its diagnosis carries great significance because it is a potentially curable disorder that is fatal if left untreated.

\section{Acknowledgments}

We are grateful to Dr. Anderson da Costa Lino Costa (Department of Pathology, Hospital Heliópolis) for his help in preparing the photographs of the duodenal biopsies.

\section{References}

1. Relman DA, Schmidt TM, Mac Dermott RP, Falcow S. Identification of the uncultered bacillus of Whipple's disease. N Engl J Med 1992; 327: 293-301.

2. Durand DU, Lecomte C, Cathébras P, Rousset H, Godeau P. SNFMI research group. Whipple's disease: clinical review of 52 cases. Medicine 1997; 76: 170-84.

3. Dobbins WO III. The diagnosis of Whipple's disease. NEngl J Med 1995; 332: 390-2.

4. Müller C, Stain C, Burghuber O. Tropheryma Whippelli in pheripheral blood mononuclear cells and cells pleural effusion. Lancet 1993; 341:701.
5. Angelini P, Trivelloto M, Donis J, Leachman RD. Myocardial bridges: a review. Prog Cardiovasc Dis 1983; 26: 75-88.

6. Thauth J, Sullebarger T. Myocardial infarction associated with myocardial bridging: case history and review of the literature. Cath Cardiovasc Diag 1997; 40: 364-7.

7. Cutler D, Wallace JM. Myocardial bridging in a young patient with sudden death. Clin Cardiol 1997; 20: 581-3. 
8. Upton AC. Histochemical investigation of mesenchymal lesions in Whipple's disease. Am J Clin Pathol 1952; 22: 755-64.

9. Silvestry FE, Kim B, Pollack BJ, et al. Cardiac Whipple disease: identification of Whipple bacillus by electron microscopy of a patient before death. Ann Intern Med 1997; 126: 214-6.

10. Khairy P, Graham AF. Whipple disease and the heart. Can J Cardiol 1996; 12: 831-4.
11. Ferrari ML, Vilela EG, Faria LC, et al. Whipple's disease: report of five cases with different clinical features. Rev Inst Med Trop S Paulo 2001; 43: 45-50.

12. Jeserich M, Ihling C, Holubarsch C. Aortic valve endocarditis with Wipple disease [letter]. Ann Intern Med 1997; 126: 920.

13. James TN, Bukley BH. Abnormalities of the coronary arteries in Whipple's disease. Am Heart J 1983; 105: 481-491. 